\title{
Enhancing the Speed and Sensitivity of a Nonlinear Optical Sensor with Noise
}

\author{
Said R.K. Rodriguez $\oplus^{*}$ \\ Center for Nanophotonics, AMOLF, Science Park 104, 1098 XG Amsterdam, Netherlands
}

(Received 15 August 2019; revised manuscript received 18 December 2019; accepted 14 January 2020; published 13 February 2020)

\begin{abstract}
We demonstrate how noise can be turned into a resource for optical sensing using a nonlinear cavity. The cavity is driven by a continuous-wave laser into the regime of optical bistability. Due to the influence of fluctuations, the cavity switches randomly between two metastable states. By analyzing the residence times in these two states, perturbations to the resonance frequency of the cavity can be detected. Here, such an analysis is presented as a function of the strength of the perturbation and of the noise. By increasing the standard deviation of the noise, we find that the detection speed increases monotonically while the sensitivity peaks at a finite value of the noise strength. Furthermore, we discuss how noise-assisted sensing can be optimized in state-of-the-art experimental platforms, relying solely on the minimum amount of noise present in the cavity due to its dissipation. These results open up new perspectives for the ultrafast detection of nanoparticles, contaminants, gases, or other perturbations to the resonance frequency of an optical resonator, at low powers and in noisy environments.
\end{abstract}

DOI: 10.1103/PhysRevApplied.13.024032

\section{INTRODUCTION}

A sensor is a device that reports a change in its environment. The sensor-environment coupling leads to dissipation, which - according to the fluctuationdissipation theorem [1] - makes the output of the sensor necessarily noisy. This minimum amount of noise places a lower bound on the magnitude of the perturbation that a linear dissipative sensor can detect within a certain time. Additional noise in the sensor or the environment typically degrades the sensing performance further; the measurement time needed to detect a certain perturbation only increases with the noise strength.

In 2002, Gammaitoni and Bulsara introduced a sensor the performance of which can be enhanced by nonlinearity and noise; they called it a noise-activated nonlinear dynamical sensor (NANDS) [2]. The physics of a NANDS is reminiscent of the Brownian particle in a double-well potential (DWP) mastered by Kramers [3]. If the DWP is symmetric, the average residence time of the particle in each well is the same. However, if the DWP is tilted, the residence-time difference (RTD) is nonzero on average. Thus, RTD measurements can be used to detect perturbations affecting the symmetry of the

*s.rodriguez@amolf.nl

Published by the American Physical Society under the terms of the Creative Commons Attribution 4.0 International license. Further distribution of this work must maintain attribution to the author(s) and the published article's title, journal citation, and DOI. potential. A similar sensing scheme can be realized with noisy nonlinear oscillators. A cubic nonlinearity leads to an effective DWP and noise can make the oscillator switch between two states corresponding to the minima of the DWP [4].

Up to now, the RTD sensing scheme has been successfully employed in the context of magnetic field detection $[5,6]$. Experiments and calculations on NANDS have focused on configurations involving a periodic modulation of the DWP [5-10], where noise plays a secondary role with respect to the periodic force. This is likely the best detection strategy in systems where slow dynamics and weak noise make fully noise-activated sensing too slow or impractical. As shown later, the situation is different for several technologically relevant optical systems.

Linear optical resonators are already well known for their sensing capabilities, which are mainly attributed to their high quality factors, small mode volumes, high operation frequencies, and the possibility of easily and remotely reading out their state with light [11-20]. Nonetheless, as the size and power budget of optical sensors continue to decrease, noise is playing an increasingly deleterious role in their performance. In this vein, many efforts have focused on realizing nanophotonic sensors with enhanced sensitivities, as expected near exceptional points, for example [21-23]. However, as long as those sensors remain linear, time-invariant, and passive, noise stands on the way of exploiting the enhanced sensitivity to detect small perturbations [24-26]. These developments suggest that a detection strategy that harnesses rather than avoids noise, as in the RTD scheme, may lead to a new frontier in optical sensing. 
Here, we demonstrate how the minimum amount of noise present in a nonlinear optical resonator, as dictated by the fluctuation-dissipation theorem, can be turned into a resource for sensing. The sensing scheme that we propose is based on measuring the RTD without any periodic driving. This scheme can be realized in resonators supporting optical bistability [27-33], i.e., two steady states with different photon numbers at a single driving condition. We show that optically bistable resonators can be used as sensors with the following remarkable properties: (i) a detection speed that increases monotonically with the standard deviation of the noise and (ii) a sensitivity that is maximized for a particular value of the standard deviation of the noise.

\section{A NOISY NONLINEAR OPTICAL CAVITY AS A SENSOR}

For concreteness but without loss of generality, we consider a single-mode Fabry-Pérot cavity (see Fig. 1) as our resonator and sensor. The cavity is made of two distributed Bragg reflectors facing each other. One of the mirrors is concave, as experimentally realized in Refs. [34-36], for example. The other mirror is planar and is coated with a nonlinear material, leading to optical bistability. Singlemode operation is possible when the radius of curvature of the concave mirror and the resonance line widths are sufficiently small for the transverse cavity modes to be well isolated from each other. In the following, we investigate the dynamics of such a single-mode nonlinear cavity when its resonance frequency is perturbed. The perturbation can be a single nanoparticle entering the cavity's mode volume, as investigated in Ref. [35], for example.

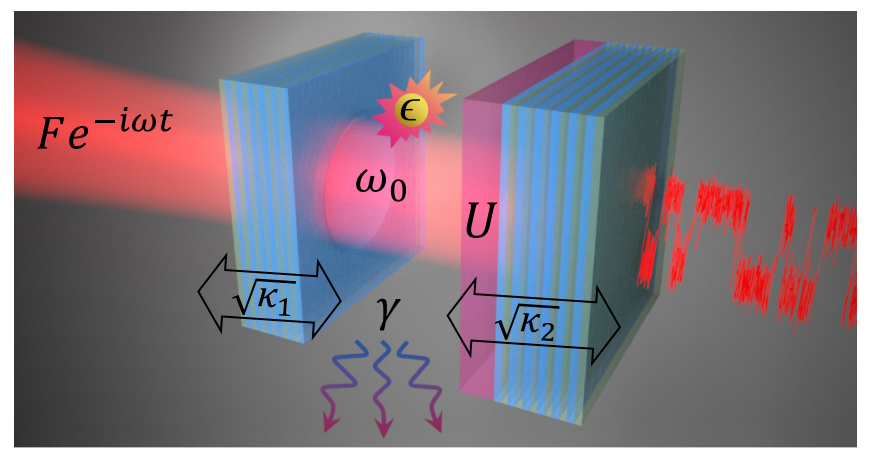

FIG. 1. A single-mode cavity with resonance frequency $\omega_{0}$, intrinsic loss rate $\gamma$, and Kerr nonlinearity corresponding to effective photon-photon interactions of strength $U$ is driven by a coherent field of amplitude $F$ and frequency $\omega . \kappa_{1,2}$ are the leakage rates of the optical mode through the mirrors. The transmitted intensity shows that the cavity switches randomly between two states due to the nonlinearity and the influence of fluctuations. The residence times of the cavity in these two states are highly sensitive to perturbations to the resonance frequency. A nanoparticle is an example of such a perturbation, here labeled as $\epsilon$.
The cavity is driven by a continuous-wave laser with frequency $\omega$ and amplitude $F$. In a frame rotating at the driving frequency, the dynamics of the intracavity field $\alpha$ are governed by the following equation:

$$
i \dot{\alpha}=\left(-\Delta-i \frac{\Gamma}{2}+U\left(|\alpha|^{2}-1\right)\right) \alpha+i \sqrt{\kappa_{1}} F+D \xi(t) .
$$

$\Delta=\omega-\omega_{0}$ is the detuning between the laser frequency and the cavity resonance frequency $\omega_{0} . \Gamma=\gamma+\kappa_{1}+\kappa_{2}$ is the total loss rate, with $\gamma$ the internal cavity loss rate and $\kappa_{1,2}$ the leakage rates of the cavity field across the mirrors. $U$ is the effective photon-photon interaction strength, associated with the nonlinear material inside the cavity. The term $D \xi(t)$ accounts for white noise, with standard deviation $D$, in the two quadratures of the light field. The stochastic term $\xi(t)=\xi(t)^{\prime}+i \xi(t)^{\prime \prime}$ is a complex Gaussian process with zero mean and is delta correlated, i.e., $\left\langle\xi^{\prime}\right\rangle=\left\langle\xi^{\prime \prime}\right\rangle=0$ and $\left\langle\xi^{\prime}(t) \xi^{\prime}\left(t+t^{\prime}\right)\right\rangle=\left\langle\xi^{\prime \prime}(t) \xi^{\prime \prime}\left(t+t^{\prime}\right)\right\rangle=$ $\delta\left(t^{\prime}\right)$. Moreover, $\xi^{\prime}$ and $\xi^{\prime \prime}$ are mutually delta correlated: $\left\langle\xi^{\prime}(t) \xi^{\prime \prime}\left(t+t^{\prime}\right)\right\rangle=\delta\left(t^{\prime}\right)$.

Let us briefly review the steady-state response of the cavity without noise, obtained by setting $\dot{\alpha}=0$ and $D=0$ in Eq. (1). Appendix A describes how to calculate the number of photons in the cavity $|\alpha|^{2}$ in steady state and how to assess the stability of those states. Figure 2(a) shows calculations of $|\alpha|^{2}$ as a function of $F$ for two different detunings $\Delta / \Gamma$; A MATLAB script producing these results is provided as Supplemental Material [37]. For $\Delta / \Gamma=1.0965$, the thick black curves represent stable states and the gray dashed curve represents unstable states. The calculation shows that there exists a range of $F$ for which two stable steady states with different photon numbers can be observed at a single driving condition. This phenomenon, known as optical bistability [27], can be observed whenever $\Delta>\sqrt{3} \Gamma / 2$, provided that $U>0$. In the bistability, the cavity can reside in either of two steady states depending on the initial conditions and the driving history of the system. Since there is no noise, the residence time of the cavity in either state is infinite. Experimentally, optical bistability can be evidenced by measuring the transmission of a laser through the cavity as a function of the laser power. In this regard, for a given $\gamma$, the values of $\kappa_{1}$ and $\kappa_{2}$ can be judiciously selected (e.g., by varying the reflectivity of the mirrors) in order to maximize the cavity transmission [38]. Here, we follow this approach by selecting $\kappa_{1}-\kappa_{2}=\gamma$. While such an enhanced transmission can be convenient for experimental implementations of the sensing scheme that we present later, the choice of $\kappa_{1,2}$ is totally irrelevant to the present theoretical work.

In the presence of noise, the cavity can switch randomly between the two states of the cavity with different numbers of photons. To illustrate this switching behavior, we perform stochastic calculations using the XSPDE package [39]. 

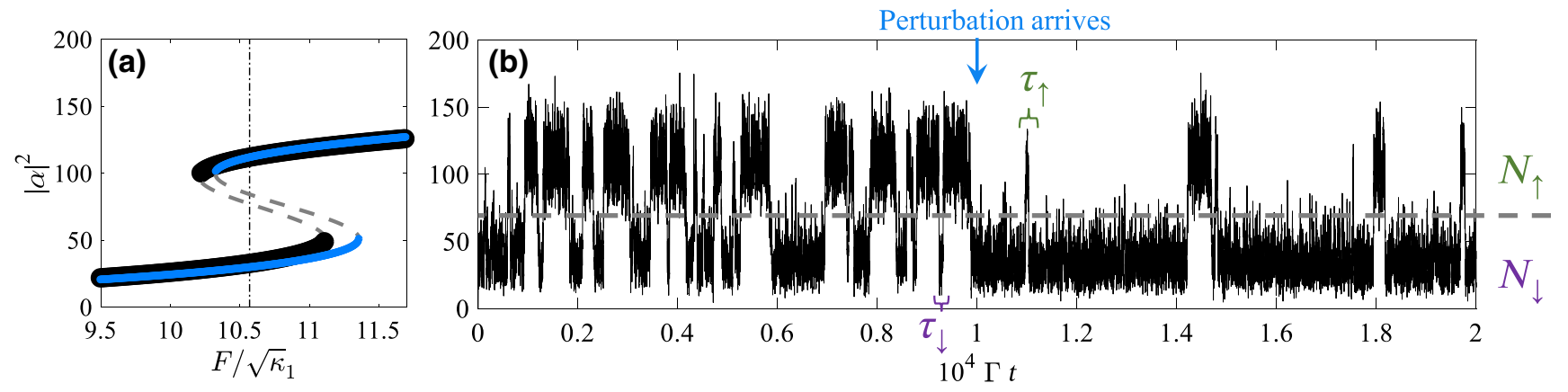

FIG. 2. (a) The steady-state number of photons in the cavity $|\alpha|^{2}$ as a function of the driving amplitude $F$ referenced to the input leakage rate $\kappa_{1}$. The solid and dashed curves correspond to stable and unstable states, respectively. The black and blue curves correspond to unperturbed and perturbed cavities. The perturbation is defined by letting the laser-cavity detuning $\Delta \rightarrow \Delta(1+\epsilon)$, with $\epsilon=0.02$. (b) The time evolution of $|\alpha|^{2}$ influenced by noise. Midway in the calculations, we introduce the aforementioned perturbation $\epsilon=0.02$. The horizontal line at $|\alpha|^{2}=69.8$ indicates the threshold above (respectively, below) which the cavity resides in state $N_{\uparrow}$ (respectively, $N_{\downarrow}$ ). Examples of the corresponding residence times are labeled as $\tau_{\uparrow}$ and $\tau_{\downarrow}$. For all calculations in Figs. 2(b), 3, 4, and 5, the values of the parameters used in the calculations are as follows: $\kappa_{1}=1 \mathrm{~s}^{-1}, \kappa_{2}=2 \kappa_{1} / 3, \Gamma=2 \kappa_{1}, F=10.57 \sqrt{\kappa_{1}}$ [indicated by the vertical dash-dotted line in (a)], $U / \Gamma=0.01, \Delta / \Gamma=1.0965$, and $D=\sqrt{\Gamma / 2}$. The same values are used for Fig. 2(a), except that $D=0$. A time step of $0.1 \Gamma^{-1}$ is used for all calculations from Fig. 2(b) onward.

In Fig. 2(b), we plot $|\alpha|^{2}$ as a function of time for constant $F=10.57 \sqrt{\kappa_{1}}, D=\sqrt{\Gamma / 2}$, and for one realization of the noise. The calculations show a bimodal distribution for $|\alpha|^{2}$ (see also Appendix B). The peaks of this distribution correspond to two attractors (stable fixed points) in phase space, associated with different average numbers of photons in the cavity. Moreover, the observed switching behavior corresponds to transitions from the basin of attraction of one stable fixed point to the basin of attraction of the other stable fixed point. In view of these dynamical effects, one could say that the states are metastable instead of steady. Metastable-state dynamics have been observed in the transmission of noisy laser-driven cavities a number of times [30-33]. Midway in the calculations in Fig. 2(b), we introduce a perturbation $\epsilon$ to the resonance frequency of the cavity. More precisely, the perturbation is set by letting $\Delta \rightarrow \Delta(1+\epsilon)$, with $\epsilon=0.02$. Since $\Delta / \Gamma$ is of order one, $\epsilon$ can be approximately interpreted as the fractional change in the resonance frequency relative to the line width. Note in Fig. 2(b) how the perturbation influences the switching behavior and biases the system toward the low-density metastable state. This biasing can be regarded as a tilting of the effective DWP for the intracavity light field. For reference, the thin blue curve in Fig. 2(a) shows the steady-state number of photons in the perturbed cavity. The biasing of the system toward the low-density state can be inferred from the enhanced proximity of the driving amplitude [vertical dashed line in Fig. 2(a)] to the threshold value for which the system jumps from the upper to the lower branch.

Similarities between light confined in a nonlinear cavity and a Brownian particle in a DWP can be recognized in the probability distribution of the complex field $\alpha$. To calculate such a distribution, we let the system evolve for a long time and plot a histogram of the state of the system as a function of the real and imaginary parts of $\alpha$. By "long time," we mean that $t v \gg 1$, with $v$ the average rate at which the cavity switches between states. For large photon numbers $\left(|\alpha|^{2} \gg 1\right)$ in the bistability region, such a histogram corresponds to the Wigner function obtained through a quantum approach [40,41].

The results of our calculations are shown in Fig. 3(a) for $\epsilon=0$ and in Fig. 3(b) for $\epsilon=0.02$. The values of the model parameters used for the calculations in Figs. 3(a) and 3(b) are the same as in the first and second halves of the time evolution in Fig. 2(b), respectively. Both panels in Fig. 3 display a bimodal distribution indicating bistability. The peaks of these distributions represent the minima of the DWP in Kramers' problem. Note, however, that the effective potential for the light field involves two dynamical variables, namely the real and imaginary parts of $\alpha$. Comparison of Figs. 3(a) and 3(b) reveals that the perturbation decreases the probability of finding the system in the state around $\operatorname{Re}[\alpha]=10.5$ and $\operatorname{Im}[\alpha]=0$, which is the high-density state. This is akin to tilting the DWP toward the low-density state. Note also that the highdensity state has a significantly reduced uncertainty along the real component of $\alpha$. This squeezing may be exploited for sensing by performing homodyne detection, but we will not comment on this further.

\section{SENSITIVITY AND DETECTION SPEED}

We would now like to quantify the sensitivity of the cavity to perturbations in $\Delta$. Such perturbations are common in optical sensing settings such as the one illustrated in Fig. 1. Note, however, that the stochastic dynamics of the cavity actually depend on the ratio $\Delta / \Gamma[32]$. Here, for 


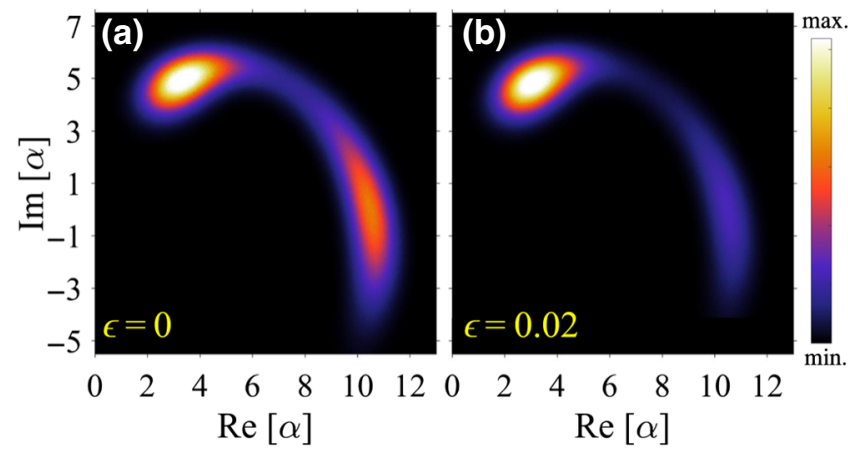

FIG. 3. The probability distribution of the complex field $\alpha$ for the (a) unperturbed and (b) perturbed cavity. The perturbation corresponds to letting $\Delta \rightarrow \Delta(1+\epsilon)$, with $\epsilon=0.02$. Other values of parameters used in the calculations are given in Fig. 2.

simplicity, we assume that $\Gamma$ is unaffected by the perturbation. In the event that such an assumption cannot be made, perturbations that increase both $\Delta$ and $\Gamma$ can be regarded as effectively weaker than those that increase only $\Delta$ or $\Gamma$.

The first step in our sensing protocol is to define a threshold density $N_{\text {th }}$ above (respectively, below) which the cavity is said to reside in state $N_{\uparrow}$ (respectively, $N_{\downarrow}$ ). Appendix B shows how $N_{\text {th }}$ can be defined by analyzing the probability density function of $|\alpha|^{2}$. Next, we define residence times $\tau_{\uparrow}$ and $\tau_{\downarrow}$ as the time intervals for which the cavity continuously resides in states $N_{\uparrow}$ and $N_{\downarrow}$, respectively. For example, in Fig. 2(b), we indicate two events with residence times $\tau_{\uparrow}$ and $\tau_{\downarrow}$ and we indicate $N_{\text {th }}$ with a horizontal dashed line. Naturally, the random nature of the switching leads to distributions of residence times. Therefore, a reliable detection strategy should be constructed based on the properties of these distributions. In particular, we inspect the time-averaged RTD $\delta \tau=\tau_{\uparrow}-\tau_{\downarrow}$.

In Fig. 4, we analyze how $\delta \tau$ (referenced to the constant $\left.\bar{\tau}_{0}=\left[\tau_{\uparrow}(\epsilon=0)+\tau_{\downarrow}(\epsilon=0)\right] / 2\right)$ scales with the strength of the perturbation $\epsilon$. The open circles are the result of numerical calculations for the same parameter values as in Fig. 2(b) and Fig. 3, except that $\epsilon$ is now varied. For all $\epsilon, \delta \tau$ is first time averaged based on 4000 residence events and then ensemble averaged over 100 realizations with different noise seeds. By varying the noise seed, we take into account that nominally identical measurements can give slightly different values of $\delta \tau$ in finite time. Most importantly, by varying the noise seed we avoid having the same realization of the noise for any two perturbations; that would enable the detection of arbitrarily small perturbations because the stochastic trajectories of cavity fields with different perturbations would be correlated.

Figure 4 shows that $\delta \tau$ scales nonlinearly with $\epsilon$. This scaling behavior can be understood by recalling the aforementioned analogy between a noisy Kerr-nonlinear cavity and a Brownian particle in a DWP. As shown by Kramers

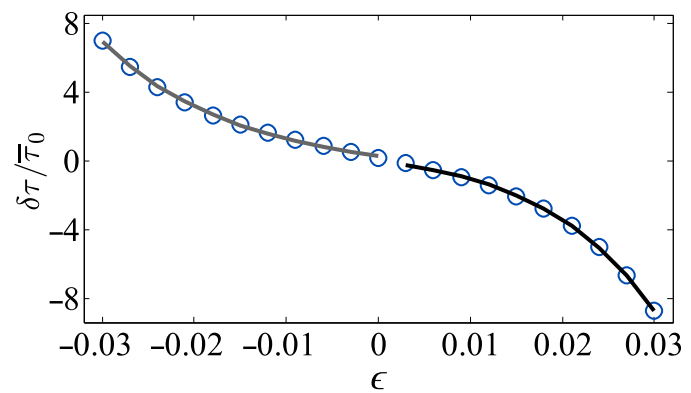

FIG. 4. The average RTD $\delta \tau$ between the $N_{\uparrow}$ and $N_{\downarrow}$ states (see Fig. 2) versus the strength of the perturbation $\epsilon$ in the detuning. $\delta \tau$ is divided by $\bar{\tau}_{0}=\left[\tau_{\uparrow}(\epsilon=0)+\tau_{\downarrow}(\epsilon=0)\right] / 2$, which is the average residence time of the system in states $N_{\uparrow}$ and $N_{\downarrow}$ at $\epsilon=$ 0 . In this way, changes in $\delta \tau$ relative to the residence times of the unperturbed cavity can be estimated. The gray and black curves are exponential fits to the data as explained in the text. The values of the parameters used in the calculations are given in Fig. 2.

[3], the average residence time of the Brownian particle in one well grows exponentially with the ratio of the barrier height to the thermal energy. In our noisy nonlinear optical cavity, the effective barrier height is determined by $\epsilon$, which can tilt the DWP as shown in Fig. 3. Therefore, we may expect an exponential scaling of $\delta \tau$ with $\epsilon$. To verify this hypothesis, we fit the numerical results in Fig. 4 with a function of the form $A e^{B \epsilon}+C$, with $A, B$, and $C$ as the fitting parameters. For this purpose, the numerical data are separated into two parts. One part is associated with $\delta \tau \geq 0$ and the other part is associated with $\delta \tau \leq 0$. This partition is made because the output of the fitting function is strictly non-negative and for the second part $\delta \tau \leq 0$. Thus, since we are only interested in the scaling behavior, we let $\delta \tau \rightarrow-\delta \tau$ for the second part. Figure 4 shows as a gray curve the fit to the part of the data for which $\delta \tau \geq 0$ and as a black curve the fit to the part of the data for which $\delta \tau \leq 0$. In both cases, the high quality of the exponential fits to the numerical data confirms the following: the signal of our sensor, namely $\delta \tau$, scales exponentially with the strength of the perturbation $\epsilon$. This result stands in stark contrast with the behavior of standard optical sensors, for which the signal (e.g., the change in the number of photons in the cavity) scales linearly with the perturbation. Therefore, relatively large perturbations can be much more easily detected with our nonlinear sensing scheme. Note, however, that $\delta \tau$ is approximately linear for small $\epsilon$. Hence, we can fit a line to the data near $\epsilon=0$ in order to estimate a lower bound of the sensitivity $\mathcal{S}=\partial \delta \tau / \partial \epsilon$. From the slope of the fitted line (not shown), we obtain the lower bound $\mathcal{S}_{\mathcal{L B}}=138.6 \pm 9 \mathrm{~s}$, with the uncertainty corresponding to a $95 \%$ confidence interval.

Another important figure of merit is the detection speed. Assessing the detection speed of our stochastic nonlinear cavity is tantamount to answering the following question: how many residence events are needed to detect a certain 


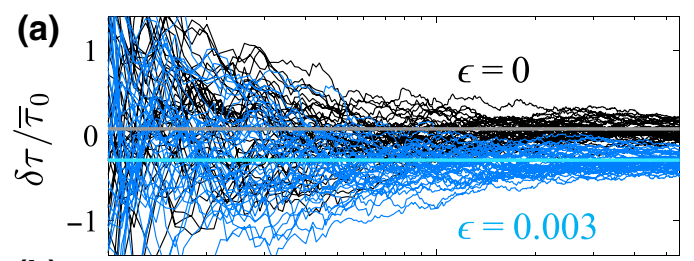

(b)

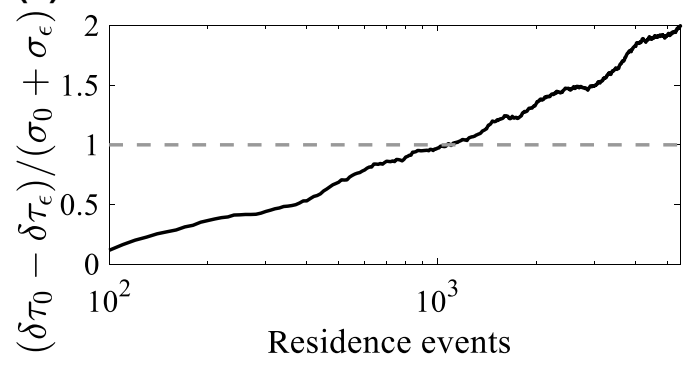

FIG. 5. (a) The average RTD, as in Fig. 4, as a function of the number of residence events over which the averaging is performed. The black and blue lines correspond to unperturbed and perturbed cavities, respectively. Different lines of the same color correspond to different realizations of the noise. The thick horizontal gray and blue lines indicate the long-time average RTD for the unperturbed and perturbed cavities, respectively. (b) $\delta \tau_{0}$ and $\delta \tau_{\epsilon}$ are the average RTD without and with the perturbation, respectively. $\sigma_{0}$ and $\sigma_{\epsilon}$ are standard deviations of the RTD distributions without and with the perturbation, respectively. Each element in a RTD distribution is associated with a different noise seed in the calculation. $\left(\delta \tau_{0}-\delta \tau_{\epsilon}\right) /\left(\sigma_{0}+\sigma_{\epsilon}\right)>1$, indicated by the dashed gray line, can be considered as the detection threshold. The values of the parameters used in the calculations are given in Fig. 2.

perturbation? In Fig. 5, we illustrate how this question can be answered, taking a tiny perturbation of $\epsilon=0.003$ as an example. Figure 5(a) shows several calculations of $\delta \tau$ as the number of residence events involved in the timeaveraging increases. The black and blue lines correspond to unperturbed $(\epsilon=0)$ and perturbed $(\epsilon=0.003)$ cavities, respectively. Various lines of the same color correspond to nominally identical configurations but with different realizations of the noise $\xi(t)$ obtained from different seeds. The results in Fig. 5(a) show how, as the number of residence events involved in the time averaging increases, the spread in the values of $\delta \tau$ decreases. For $t \rightarrow \infty$, all values of $\delta \tau$ for a given $\epsilon$ converge to a single value indicated by a thick horizontal line. The long-time difference in $\delta \tau$ with and without perturbation is determined by the aforementioned sensitivity.

The number of residence events needed to detect $\epsilon=$ 0.003 with a single noise realization can be determined by comparing two quantities: (i) the change in the average RTD due to the perturbation, i.e., $\delta \tau_{0}-\delta \tau_{\epsilon}$; and (ii) the sum of the standard deviations of the RTD distributions, i.e., $\sigma+\sigma_{\epsilon}$. We recall that each element of the RTD distributions is associated with a different noise seed. A simple criterion for detection can be $\left|\delta \tau_{0}-\delta \tau_{\epsilon}\right|>\left(\sigma_{0}+\sigma_{\epsilon}\right)$. This corresponds to a shift in $\delta \tau$ that is greater than the sum of the uncertainties. Using this criterion, Fig. 5(b) shows that $\epsilon=0.003$ can be detected with approximately 1000 residence events or more. Note that we adopt a stringent criterion for the detection threshold. A reliable detection strategy can still be constructed with significantly fewer residence events, provided that we accept larger probabilities of false alarm and missed detection [42].

In the following, we show how the sensing performance of the cavity depends on the standard deviation of the noise $D$. To this end, we vary $D$ without adjusting $\Gamma$. This enables us to demonstrate that the minimum amount of noise imposed by the fluctuation-dissipation relation is sufficient to enhance the performance of the sensor. Otherwise, if we were to adjust $\Gamma$ according to $D$ in order to always satisfy the fluctuation-dissipation relation, the laser amplitude $F$ needed for bistability would change. Consequently, the number of cavity photons in the metastable states would also change. All of these changes would make it difficult to reveal how the sensing performance depends on the noise. Hence, we take a pragmatic approach and vary $D$ alone, with the understanding that: (i) $D>\sqrt{\Gamma / 2}$ simply corresponds to a physical system with more noise than that imposed by the fluctuation-dissipation relation; and (ii) while $D<\sqrt{\Gamma / 2}$ is not physically possible, it is a convenient theoretical parameter regime (widely used to describe deterministic systems) allowing us to reveal the minimum amount of noise needed for our detection strategy to succeed.

In Fig. 6, we plot the average residence time of the unperturbed cavity in the two states, $\bar{\tau}_{0}=\left[\tau_{\uparrow}(\epsilon=0)+\right.$ $\left.\tau_{\downarrow}(\epsilon=0)\right] / 2$ as a function of $D / \sqrt{\Gamma / 2}$. The plot shows how the number of residence events within a fixed measurement time increases with $D$. Note that $\bar{\tau}_{0}$ decreases by approximately 3 orders of magnitude within a factor of 2.5 increase in $D / \sqrt{\Gamma / 2}$. This result, together with the result in Fig. 5(b), seems to suggest that the sensing performance of our nonlinear cavity improves indefinitely with the addition of noise. However, this is not the case because the sensitivity also depends on the noise.

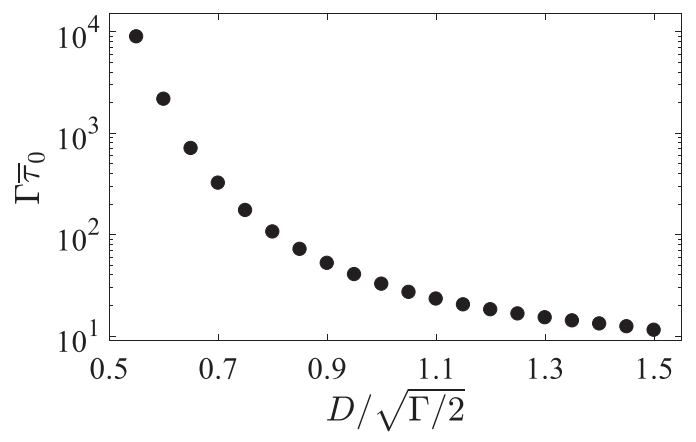

FIG. 6. The average residence time for $\epsilon=0, \bar{\tau}_{0}$, times the total loss rate $\Gamma$, as a function of the standard deviation of the noise $D$ referenced to $\sqrt{\Gamma / 2}$. 


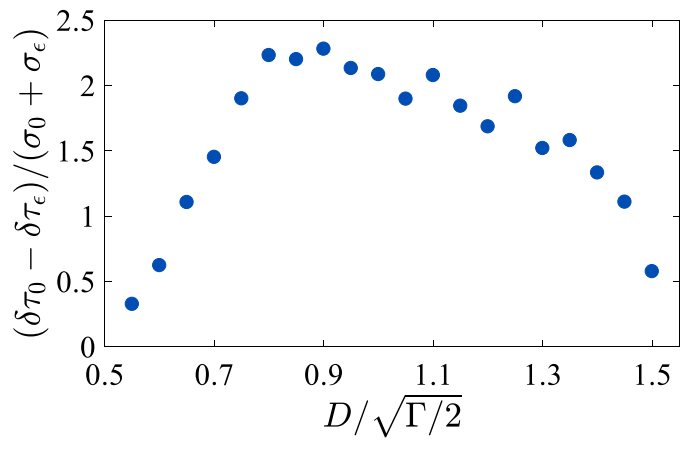

FIG. 7. The same as in Fig. 5(b), but now as a function of $D / \sqrt{\Gamma / 2}$. Other values of parameters used in the calculations are the same as in Fig. 2.

In Fig. 7, we plot $\left(\delta \tau_{0}-\delta \tau_{\epsilon}\right) /\left(\sigma_{0}+\sigma_{\epsilon}\right)$ as a function of $D / \sqrt{\Gamma / 2}$, for a fixed measurement time $2 \times 10^{5} \Gamma^{-1}$. As before, we take $\epsilon=0.003$ as an example. Recall that $\left|\delta \tau_{0}-\delta \tau_{\epsilon}\right|$ is proportional to the sensitivity and $\mid \delta \tau_{0}-$ $\delta \tau_{\epsilon} \mid>\left(\sigma_{0}+\sigma_{\epsilon}\right)$ is the detection threshold we previously defined. According to the results in Fig. 7, $\epsilon=0.003$ can only be detected within a finite range of nonzero noise. For the parameter values that we choose (achievable with modern semiconductor cavities [31-33], for example), the sensitivity is greatest for $D \approx 0.9 \sqrt{\Gamma / 2} . D=\sqrt{\Gamma / 2}$ is exactly the minimum amount of noise in the cavity demanded by the fluctuation-dissipation relation. Moreover, for $D=\sqrt{\Gamma / 2}$, the average residence time at the center of the bistability, where $\tau_{\uparrow}=\tau_{\downarrow}$, corresponds to the so-called quantum-tunneling time of bistability [32]. Details on how to calculate this tunneling time based on a quantum-master-equation approach can be found in Refs. $[41,43]$.

To understand why the sensitivity peaks for a finite amount of noise, consider the behavior of the system for extreme values of $D$. For $D \rightarrow 0$, the number of residence events within the measurement time decreases. Consequently, $\sigma+\sigma_{\epsilon}$ increases and the presence of a small perturbation becomes increasingly uncertain. Conversely, for large $D$ the residence time becomes too short. Effectively, this can be associated with a potential barrier that is too small. Such a small barrier makes it practically impossible to detect perturbations affecting the symmetry of the potential. In particular, the sensitivity is degraded when the height of the barrier is much less than (i) the change in energy between the minima of the DWP due to the perturbation and (ii) the average energy in the fluctuations. Hence, a finite amount of noise is needed for a detection strategy based on RTDs to succeed.

In practice, a system can be operated with the optimum amount of noise either by injecting noise or by judiciously selecting the laser intensity and the laser-cavity detuning. The laser power and detuning determine the number of photons involved in the bistability, which in turn determines the average residence times. Thus, by varying the laser parameters one can effectively adjust the potential barrier. Note also that the overall optimum amount of noise does not need to coincide with the peak sensitivity. For instance, the results in Figs. 6 and 7 show that increasing $D / \sqrt{\Gamma / 2}$ from 0.8 to 1.2 degrades the RTD shift by approximately $7 \%$, while the number of residence events that can be acquired within a fixed measurement time increases by approximately $460 \%$. Correspondingly, the amount of noise optimizing the overall performance of the sensor is above the value for which the sensitivity peaks.

In order to connect our proposal with a potential experimental realization, we would like to specify parameter values that may be achieved using present-day technologies. For example, consider a cavity with a resonance frequency $\omega_{0}=360 \mathrm{THz}$, a total loss rate $\Gamma=10 \mathrm{GHz}$, and $U / \Gamma=0.01$; these values are typical for III-V semiconductor cavities in Refs. [32,33,44,45]. The calculations in Figs. 2-7 are all done for $U / \Gamma=0.01$, so we can immediately read out the corresponding time scales. In particular, in Fig. 5 we note that a perturbation of $\epsilon=0.003$ can be detected with approximately 1000 residence events, using the minimum amount of noise present in the cavity due to the dissipation. For a switching rate of $10 \gamma$, which can be achieved in the range $0.9 \gtrsim \Delta / \Gamma \gtrsim 1$, we conclude that a tiny shift in the resonance frequency that is approximately $0.3 \%$ of the line width can be detected within a measurement time of approximately $10 \mathrm{~ns}$. Larger perturbations (still on the order of a few percent of the resonance line width) that make the system depart out of the bistability can be detected with fewer residence events, since the system will stop switching. For the parameter values we consider, this means that perturbations of a few percent of the resonance line width can be detected within approximately $0.1 \mathrm{~ns}$.

In our analysis so far, we have not mentioned the fact that the residence times also depend on the strength of the photon-photon interactions relative to the dissipation, i.e., $U / \Gamma$ [32]. As $U / \Gamma$ decreases, the number of photons involved in the bistability increases. Consequently, residence times for fixed $\Delta / \Gamma$ will be longer at the center of the bistability. This is not a problem for our sensing scheme, as long as greater laser power is available and the frequency of the laser or the cavity resonance can be tuned. As shown in Ref. [32], for small detunings $(\Delta / \Gamma \sim 0.9)$ residence times at the center of the bistability are all on the order of $10 \Gamma^{-1}$ for vastly different values of $U / \Gamma$. Thus, our sensing scheme can be realized in systems with vastly different, albeit finite, nonlinearity. The validity of our model is only expected to break down for extremely strong nonlinearity, i.e., $U / \Gamma \sim 1$. In those cases, our mean-field equation plus stochastic terms (the so-called truncated Wigner approximation [46]) is no longer valid, and a full quantum-master-equation approach is needed. 


\section{CONCLUSION AND PERSPECTIVES}

In conclusion, we propose a noisy nonlinear optical cavity as a reliable and ultrafast sensor. Such a sensor can be used to detect perturbations to the resonance frequency of the cavity. The detection speed of this sensor increases with the noise strength, while its sensitivity peaks for a particular noise strength. This unusual dependence of the sensing performance on noise may open up new possibilities for sensing at low optical powers and in noisy environments. Noise-assisted optical sensing can be used to detect nanoparticles, contaminants, biological entities, or gases, affecting the resonance frequency of an optical resonator, for example.

For concreteness, we focus the discussion on a FabryPérot cavity. However, our results hold for any singlemode nonlinear optical resonator. For example, microdisks [17], ring resonators [28], and photonic crystal cavities [29] have all displayed optical bistability at room temperature. Alternatively, an overdamped optically levitated nanoparticle [47] can also display bistability. These systems are therefore suitable candidates for realizing the sensing scheme in this proposal. One should note, however, that in some of these systems bistability emerges from a slow nonlinear response - due, for example, to thermal effects. In those cases, the sensing scheme proposed herein can still be realized but the noninstantaneous character of the nonlinearity [48] will limit the operation speed of the sensor.

Beyond single-mode physics, our approach could also be implemented in systems involving two or more modes. Such systems could display regimes with greater sensitivity than single-mode systems. For example, one could drive a coupled-cavity system, as demonstrated in Ref. [49], into parametrically unstable [50,51] or symmetry-breaking $[52,53]$ regimes. Optomechanical systems involving coupled optical and mechanical modes constitute yet another interesting possibility for noise-assisted sensing. This possibility is appealing given the great current interest in optomechanical systems and the known equivalence between optomechanical systems and Kerr nonlinear resonators [54,55]. Finally, parametric driving of a nonlinear oscillator [56] is another interesting possibility that could be combined with the RTD strategy to achieve greater sensitivity.

\section{ACKNOWLEDGMENTS}

I am grateful to Javier del Pino for critical comments and to Zhou Geng and Kevin Peters for stimulating discussions. This work is part of the research program of the Netherlands Organisation for Scientific Research (NWO). I acknowledge financial support from NWO through a Veni Grant No. 016.Veni.189.039.

\section{APPENDIX A: STEADY STATES AND STABILITY} ANALYSIS

We calculate the steady-state solutions to Eq. (1) with $D=0$ by setting $\dot{\alpha}=0$. Next, we multiply the resultant equation with its complex conjugate and obtain

$$
\begin{aligned}
0= & N\left(-\Delta-i \frac{\Gamma}{2}+U(N-1)\right)\left(-\Delta+i \frac{\Gamma}{2}+U(N-1)\right) \\
& -\kappa_{1}|F|^{2},
\end{aligned}
$$

where $N=|\alpha|^{2}$ is the mean number of photons in the cavity. Equation (A1) is a third-order polynomial in $N$. Hence, we may expect three solutions corresponding to the roots of that polynomial. However, because of the requirement that $N$ be real, not all solutions may be physical. In fact, one finds that the system may support either one or three steady states depending on the parameters $(\Delta, \Gamma, U$, and $F$ ).

Having found the mean number of cavity photons in steady state, $N_{\mathrm{SS}}$, we proceed to assess the stability of those states as done, for example, in Ref. [57]. For this purpose, we first need an expression for the complex steady-state field $\alpha_{\text {SS }}$. Such an expression can be obtained by recalling the relation $\alpha=\sqrt{N} e^{i \phi}$, where $\phi$ is the phase of the intracavity field. Replacing $\alpha$ with $\sqrt{N} e^{i \phi}$ in Eq. (1) and setting $\dot{\alpha}=0$ and $D=0$, we obtain

$$
\sqrt{N} e^{i \phi}=\frac{-i \sqrt{\kappa_{1}} F}{-\Delta-i \frac{\Gamma}{2}+U(N-1)} .
$$

Thus, we can construct $\alpha_{\mathrm{SS}}=\sqrt{N_{\mathrm{SS}}} e^{i \phi_{\mathrm{SS}}}$ by first finding the real roots of Eq. (A1) to get $N_{\mathrm{SS}}$ and then inserting $N_{\mathrm{SS}}$ in Eq. (A2) to obtain $e^{i \phi_{\mathrm{SS}}}$

We are now in a position to assess the stability of the steady states corresponding to the fields $\alpha_{\mathrm{SS}}$. To this end, we consider the dynamical effect of adding a small fluctuation $\delta \alpha$ to $\alpha_{\mathrm{SS}}$, i.e.,

$$
\tilde{\alpha}(t)=\alpha_{\mathrm{SS}}+\delta \alpha .
$$

We are interested in the time evolution of the fluctuation $\delta \alpha$ and its complex conjugate $\delta \alpha^{*}$. To get the equation of motion for $\delta \alpha$, we insert the above expression for $\tilde{\alpha}$ in Eq. (1) and retain terms that are linear in $\delta \alpha$ only. Similarly, to get the equation of motion for $\delta \alpha^{*}$, we insert $\tilde{\alpha}^{*}$ in Eq. (1) and retain terms that are linear in $\delta \alpha^{*}$ only. Consequently, 
we arrive at the coupled equations

$$
\begin{aligned}
i \frac{\partial}{\partial t}\left(\begin{array}{c}
\delta \alpha \\
\delta \alpha^{*}
\end{array}\right) & \\
= & \left(\begin{array}{cc}
-\Delta-i \frac{\Gamma}{2}+2 U(N-1) & U \alpha^{2} \\
-U\left(\alpha^{*}\right)^{2} & \Delta-i \frac{\Gamma}{2}-2 U(N-1)
\end{array}\right) \\
& \times\left(\begin{array}{c}
\delta \alpha \\
\delta \alpha^{*}
\end{array}\right) .
\end{aligned}
$$

Equation (A4) is of the form

$$
i \partial_{t} \delta \boldsymbol{\alpha}=\mathbf{A} \delta \boldsymbol{\alpha}
$$

which has solutions of the form

$$
\delta \boldsymbol{\alpha}=\eta e^{-i \lambda t}
$$

where $\eta$ and $\lambda$ are the eigenvectors and eigenvalues of A, respectively. Hence, we need to solve the eigenvalue problem

$$
\mathbf{A} \delta \alpha=\lambda \delta \alpha
$$

Equation (A6) reveals that the imaginary parts of the eigenvalues $\lambda$, i.e., $\Im[\lambda]$, determine the stability of the steady state. In particular, if $\Im[\lambda]<0$ for both eigenvalues, the fluctuations decay and the steady state is stable. Otherwise, if $\Im[\lambda] \geq 0$ for at least one eigenvalue, the fluctuations grow and the steady state is unstable.

\section{APPENDIX B: DEFINITION OF $\boldsymbol{N}_{\text {th }}$}

Here, we explain how the threshold density $N_{\text {th }}$, separating the states $N_{\uparrow}$ and $N_{\downarrow}$, can be defined. To this end, we need a long trace of the transmitted intensity by the cavity, proportional to $|\alpha|^{2}$. By creating a histogram of events with different values of $|\alpha|^{2}$, we can calculate a probability density function of $|\alpha|^{2}$ as shown in Fig. 8. The values of the model parameters used in the calculations of Fig. 8 are the same as those reported in the caption of Fig. 2. The results in Fig. 8 show a bimodal distribution, corresponding to bistability. Between the two peaks there is a local minimum in $|\alpha|^{2}$. This local minimum maps to the peak of the potential barrier in the DWP description. We therefore ascribe the value of $|\alpha|^{2}$ at this minimum to $N_{\text {th }}$.

\section{APPENDIX C: COMPARISON TO LINEAR OPTICAL SENSING}

Here, we compare the sensing performance of linear and nonlinear cavities. Using a linear cavity, a perturbation $\epsilon$ can be detected by recording the transmitted intensity $T \propto N$ at a single driving condition. A scan of $\Delta$ is unnecessary, since it does not provide more information than an

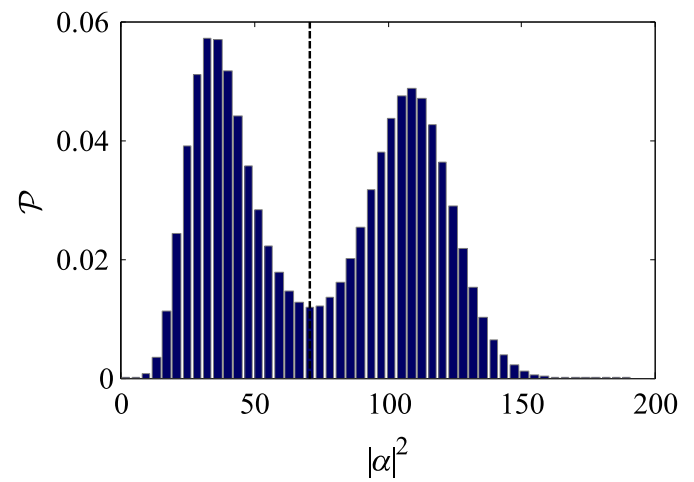

FIG. 8. The probability density function of the number of photons in the cavity, for the same parameter values as given in Fig. 2, with $\epsilon=0$. The vertical dashed line indicates a local minimum. This minimum corresponds to the threshold density $N_{\text {th }}$ above (respectively, below) which the system is said to reside in state $N_{\uparrow}$ (respectively, $N_{\downarrow}$ ).

optimal single-frequency measurement [25]. Moreover, for a sufficiently long measurement time $\tau_{m}$, there is in principle no limit to the magnitude of the perturbation that can be detected. Therefore, we are ultimately interested in the detection speed of the sensor.

To illustrate how the detection speed can be assessed in the linear case, we follow a similar approach to the one that was used to produce the results in Fig. 5. However, while for the nonlinear cavity $\delta \tau$ is the signal, for the linear cavity $N$ is the signal. To ease comparison between the two sensing schemes, we perform linear calculations using the same parameter values as used for the calculations in Fig. 5 (reported in the Fig. 2 caption), except of course $U=0$. In particular, for all calculations we set $\kappa_{1}=1 \mathrm{~s}^{-1}, \kappa_{2}=2 \kappa_{1} / 3, \Gamma=2 \kappa_{1}, F=10.57 \sqrt{\kappa_{1}}, \Delta / \Gamma=$ 1.0965 , and $D=\sqrt{\Gamma / 2}$, and we consider a perturbation of strength $\epsilon=0.003$.

Figure 9(a) shows several calculations of the photon number in the unperturbed and perturbed cavities, $N_{0}$ and $N_{\epsilon}$ respectively. $N_{0}$ and $N_{\epsilon}$ are plotted as a function of the measurement time $\tau_{m}$, which is the time over which a measurement of $N$ is averaged. The black and blue lines correspond to unperturbed $(\epsilon=0)$ and perturbed $(\epsilon=$ 0.003 ) cavities, respectively. Various lines of the same color correspond to nominally identical configurations but with different realizations of the noise $\xi(t)$ obtained from different seed. Note in Fig. 9 how the spread in the values of $N$ decreases as $\tau_{m}$ increases. As $\tau_{m} \rightarrow \infty$, all values of $N$ for a given $\epsilon$ converge to a single value indicated by a thick horizontal line.

The time needed to detect $\epsilon=0.003$ with a single noise realization can be determined by comparing the time evolution of two quantities: (i) $N_{0}-N_{\epsilon}$ averaged over different noise realizations and (ii) $\sigma+\sigma_{\epsilon}$, i.e., the sum of the 
standard deviations of $N_{0}$ and $N_{\epsilon}$. Similar to the nonlinear case, we can take $\left|N_{0}-N_{\epsilon}\right|>\left(\sigma_{0}+\sigma_{\epsilon}\right)$ as a criterion for detection. This corresponds to a shift in $N$ exceeding the sum of the uncertainties. Using this criterion, the results in Fig. 9(b) show that $\epsilon=0.003$ can be detected with $\tau_{m} \sim 15000 \Gamma^{-1}$.

Let us now compare the value of $\tau_{m}$ obtained above with the corresponding time needed to detect $\epsilon=0.003$ using a nonlinear cavity. Figure 5(b) shows that 1000 residence events are needed to detect the perturbation $\epsilon=0.003$. In addition, Fig. 6 shows that the average residence time for $\epsilon=0$ is $\bar{\tau}_{0}=32 \Gamma^{-1}$, for the particular noise strength $D=\sqrt{\Gamma / 2}$ that we take. Consequently, the measurement time needed for the nonlinear sensing scheme to succeed in this task is $\tau_{m}^{\mathrm{NL}} \sim 32000 \Gamma^{-1}$. Thus, the nonlinear cavity is approximately twice as slow as the linear cavity in detecting this small perturbation and using the minimum amount of noise imposed by fluctuation-dissipation relation.

While the above results may seem discouraging for employing the nonlinear sensing scheme, we would like to stress that the comparison has been done without

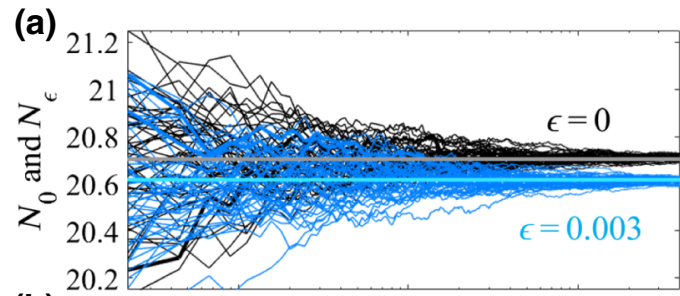

(b)

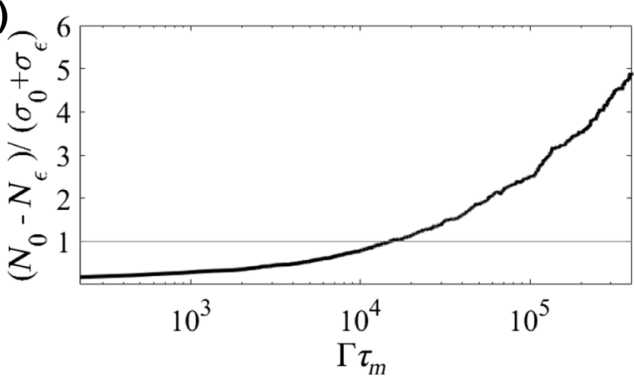

FIG. 9. (a) The average number of photons $N$ in the linear cavity $(U=0)$ as a function of the measurement time $\tau_{m}$ times the total loss rate $\Gamma$. The black and blue lines correspond to unperturbed and perturbed cavities, respectively. Different lines of the same color correspond to different realizations of the noise. The thick horizontal gray and blue lines indicate the long-time $N$ for the unperturbed and perturbed cavities, respectively. (b) $N_{0}$ and $N_{\epsilon}$ are the average number of photons without and with the perturbation, respectively. $\sigma_{0}$ and $\sigma_{\epsilon}$ are standard deviations of the number of photons without and with the perturbation, respectively. Each element in a photon-number distribution is associated with a different noise seed in the calculation. $\left(N_{0}-\right.$ $\left.N_{\epsilon}\right) /\left(\sigma_{0}+\sigma_{\epsilon}\right)>1$, indicated by the dashed gray line, can be considered as the detection threshold. The values of the parameters used in the calculations are the same as in Figs. 2(b) and 5 (given in the Fig. 2 caption), except $U=0$. optimizing parameter values. In particular, the following factors need to considered. First, the scaling of the signal $\delta \tau$ with $\epsilon$ is approximately linear for the small perturbation that we consider, as Fig. 4 shows. Therefore, we do not exploit the exponential scaling of $\delta \tau$ with $\epsilon$ in the nonlinear sensing scheme. This exponential scaling can be advantageous for greater perturbations. Second, the key feature of the nonlinear sensing scheme is its unusual dependence on noise. In particular, Fig. 6 shows that the average residence time decreases to $\bar{\tau}_{0}=15 \Gamma^{-1}$ when $D / \sqrt{\Gamma / 2}$ increases from 1.0 to 1.3 . Meanwhile, the sensitivity is only marginally affected as shown in Fig. 7. In stark contrast, for a linear optical sensor the measurement time only increases with $D$. Therefore, it is plausible that for a different set of parameters, the measurement time $\tau_{m}$ using the nonlinear cavity can be shorter than that of the linear cavity. A third point of attention is that the strength and speed of the nonlinearity, as well as noise, can be leveraged to decrease the measurement time in the nonlinear scheme. For instance, a slow (e.g., thermo-optical) nonlinearity can make residence-time distributions narrower by suppressing fast switching events. Such a high-frequency cutoff could potentially make residence-time distributions narrower, and the detection of a perturbation faster, in the nonlinear sensing scheme.

[1] R. Kubo, The fluctuation-dissipation theorem, Rep. Prog. Phys. 29, 255 (1966).

[2] L. Gammaitoni and A. R. Bulsara, Noise Activated Nonlinear Dynamic Sensors, Phys. Rev. Lett. 88, 230601 (2002).

[3] H. Kramers, Brownian motion in a field of force and the diffusion model of chemical reactions, Physica 7, 284 (1940).

[4] L. Gammaitoni, P. Hänggi, P. Jung, and F. Marchesoni, Stochastic resonance, Rev. Mod. Phys. 70, 223 (1998).

[5] B. Ando, S. Baglio, A. R. Bulsara, and V. Sacco, "Residence times difference" fluxgate magnetometers, IEEE. Sens. J. 5, 895 (2005).

[6] S. Baglio, A. R. Bulsara, B. Ando, S. La Malfa, V. Marletta, C. Trigona, P. Longhini, A. Kho, V. In, J. D. Neff, G. W. Anderson, C. C. Obra, B. K. Meadows, and A. Palacios, Exploiting nonlinear dynamics in novel measurement strategies and devices: From theory to experiments and applications, IEEE T. Instrum. Meas. 60, 667 (2011).

[7] A. R. Bulsara, C. Seberino, L. Gammaitoni, M. F. Karlsson, B. Lundqvist, and J. W. C. Robinson, Signal detection via residence-time asymmetry in noisy bistable devices, Phys. Rev. E 67, 016120 (2003).

[8] A. Nikitin, N. G. Stocks, and A. R. Bulsara, Signal detection via residence times statistics: Noise-mediated minimization of the measurement error, Phys. Rev. E 68, 036133 (2003).

[9] A. Dari, L. Bosi, and L. Gammaitoni, Nonlinear sensors: An approach to the residence time detection strategy, Phys. Rev. E 81, 011115 (2010). 
[10] A. Nikitin, N. Stocks, and A. Bulsara, Bistable sensors based on broken symmetry phenomena: The residence time difference vs. the second harmonic method, Eur. Phys. J. 222, 2583 (2013).

[11] A. D. McFarland and R. P. Van Duyne, Single silver nanoparticles as real-time optical sensors with zeptomole sensitivity, Nano Lett. 3, 1057 (2003).

[12] F. Vollmer and S. Arnold, Whispering-gallery-mode biosensing: Label-free detection down to single molecules, Nat. Methods 5, 591 (2008).

[13] J. Zhu, S. K. Ozdemir, Y.-F. Xiao, L. Li, L. He, D.-R. Chen, and L. Yang, On-chip single nanoparticle detection and sizing by mode splitting in an ultrahigh- $Q$ microresonator, Nat. Photon. 4, 46 (2010).

[14] P. Offermans, M. C. Schaafsma, S. R. K. Rodriguez, Y. Zhang, M. Crego-Calama, S. H. Brongersma, and J. Gmez Rivas, Universal scaling of the figure of merit of plasmonic sensors, ACS Nano 5, 5151 (2011).

[15] P. Zijlstra, P. M. Paulo, and M. Orrit, Optical detection of single non-absorbing molecules using the surface plasmon resonance of a gold nanorod, Nat. Nanotechnol. 7, 379 (2012).

[16] L. Shao, X.-F. Jiang, X.-C. Yu, B.-B. Li, W. R. Clements, F. Vollmer, W. Wang, Y.-F. Xiao, and Q. Gong, Detection of single nanoparticles and lentiviruses using microcavity resonance broadening, Adv. Mater. 25, 5616 (2013).

[17] M. R. Foreman, J. D. Swaim, and F. Vollmer, Whispering gallery mode sensors, Adv. Opt. Photon. 7, 168 (2015).

[18] S. Rosenblum, Y. Lovsky, L. Arazi, F. Vollmer, and B. Dayan, Cavity ring-up spectroscopy for ultrafast sensing with optical microresonators, Nat. Commun. 6, 6788 (2015)

[19] H. Kelkar, D. Wang, D. Martín-Cano, B. Hoffmann, S. Christiansen, S. Götzinger, and V. Sandoghdar, Sensing Nanoparticles with a Cantilever-Based Scannable Optical Cavity of Low Finesse and Sub- $\lambda^{3}$ Volume, Phys. Rev. Appl. 4, 054010 (2015).

[20] Y. Zhi, X.-C. Yu, Q. Gong, L. Yang, and Y.-F. Xiao, Single nanoparticle detection using optical microcavities, Adv. Mater. 29, 1604920 (2017).

[21] J. Wiersig, Enhancing the Sensitivity of Frequency and Energy Splitting Detection by Using Exceptional Points: Application to Microcavity Sensors for Single-Particle Detection, Phys. Rev. Lett. 112, 203901 (2014).

[22] W. Chen, Ş. K. Özdemir, G. Zhao, J. Wiersig, and L. Yang, Exceptional points enhance sensing in an optical microcavity, Nature 548, 192 (2017).

[23] Q. Zhong, J. Ren, M. Khajavikhan, D. N. Christodoulides, I. M. C. K. Özdemir, and R. El-Ganainy, Sensing with Exceptional Surfaces in Order to Combine Sensitivity with Robustness, Phys. Rev. Lett. 122, 153902 (2019).

[24] W. Langbein, No exceptional precision of exceptional-point sensors, Phys. Rev. A 98, 023805 (2018).

[25] H.-K. Lau and A. A. Clerk, Fundamental limits and nonreciprocal approaches in non-Hermitian quantum sensing, Nat. Commun. 9, 4320 (2018).

[26] N. A. Mortensen, P. A. D. Gonçalves, M. Khajavikhan, D. N. Christodoulides, C. Tserkezis, and C. Wolff, Fluctuations and noise-limited sensing near the exceptional point of parity-time-symmetric resonator systems, Optica 5, 1342 (2018).
[27] H. Gibbs, Optical Bistability: Controlling Light with Light, Quantum Electronics Series (Academic Press, 1985).

[28] V. R. Almeida and M. Lipson, Optical bistability on a silicon chip, Opt. Lett. 29, 2387 (2004).

[29] M. Notomi, A. Shinya, S. Mitsugi, G. Kira, E. Kuramochi, and T. Tanabe, Optical bistable switching action of Si high$Q$ photonic-crystal nanocavities, Opt. Express 13, 2678 (2005).

[30] J. Kerckhoff, M. A. Armen, and H. Mabuchi, Remnants of semiclassical bistability in the few-photon regime of cavity QED, Opt. Express 19, 24468 (2011).

[31] H. Abbaspour, G. Sallen, S. Trebaol, F. Morier-Genoud, M. T. Portella-Oberli, and B. Deveaud, Effect of a noisy driving field on a bistable polariton system, Phys. Rev. B 92, 165303 (2015).

[32] S. R. K. Rodriguez, W. Casteels, F. Storme, N. Carlon Zambon, I. Sagnes, L. Le Gratiet, E. Galopin, A. Lemaître, A. Amo, C. Ciuti, and J. Bloch, Probing a Dissipative Phase Transition via Dynamical Optical Hysteresis, Phys. Rev. Lett. 118, 247402 (2017).

[33] T. Fink, A. Schade, S. Höfling, C. Schneider, and A. Imamoglu, Signatures of a dissipative phase transition in photon correlation measurements, Nat. Phys. 14, 365 (2018).

[34] A. A. P. Trichet, J. Foster, N. E. Omori, D. James, P. R. Dolan, G. M. Hughes, C. Vallance, and J. M. Smith, Openaccess optical microcavities for lab-on-a-chip refractive index sensing, Lab Chip 14, 4244 (2014).

[35] A. A. P. Trichet, P. R. Dolan, D. James, G. M. Hughes, C. Vallance, and J. M. Smith, Nanoparticle trapping and characterization using open microcavities, Nano Lett. 16, 6172 (2016).

[36] M. H. Bitarafan and R. G. DeCorby, On-chip high-finesse Fabry-Perot microcavities for optical sensing and quantum information, Sensors 17, 1748 (2017).

[37] See the Supplemental Material at http://link.aps.org/supple mental/10.1103/PhysRevApplied.13.024032 for a MATLAB script for carrying out the calculations in Fig. 2(a).

[38] S. R. K. Rodriguez, V. Goblot, N. C. Zambon, A. Amo, and J. Bloch, Nonreciprocity and zero reflection in nonlinear cavities with tailored loss, Phys. Rev. A 99, 013851 (2019).

[39] S. Kiesewetter, R. Polkinghorne, B. Opanchuk, and P. D. Drummond, XSPDE: Extensible software for stochastic equations, SoftwareX 5, 12 (2016).

[40] K. Vogel and H. Risken, Quasiprobability distributions in dispersive optical bistability, Phys. Rev. A 39, 4675 (1989).

[41] W. Casteels, R. Fazio, and C. Ciuti, Critical dynamical properties of a first-order dissipative phase transition, Phys. Rev. A 95, 012128 (2017).

[42] S. Kay, Fundamentals of Statistical Signal Processing: Detection Theory, Prentice Hall Signal Processing Series (Prentice-Hall PTR, 1998).

[43] H. Risken, C. Savage, F. Haake, and D. F. Walls, Quantum tunneling in dispersive optical bistability, Phys. Rev. A 35, 1729 (1987).

[44] A. F. Adiyatullin, M. D. Anderson, H. Flayac, M. T. Portella-Oberli, F. Jabeen, C. Ouellet-Plamondon, G. C. Sallen, and B. Deveaud, Periodic squeezing in a polariton Josephson junction, Nat. Commun. 8, 1329 (2017). 
[45] L. Pickup, K. Kalinin, A. Askitopoulos, Z. Hatzopoulos, P. G. Savvidis, N. G. Berloff, and P. G. Lagoudakis, Optical Bistability under Nonresonant Excitation in Spinor Polariton Condensates, Phys. Rev. Lett. 120, 225301 (2018).

[46] I. Carusotto and C. Ciuti, Quantum fluids of light, Rev. Mod. Phys. 85, 299 (2013).

[47] F. Ricci, R. A. Rica, M. Spasenović, J. Gieseler, L. Rondin, L. Novotny, and R. Quidant, Optically levitated nanoparticle as a model system for stochastic bistable dynamics, Nat. Commun. 8, 15141 (2017).

[48] Z. Geng, K. J. H. Peters, A. A. P. Trichet, K. Malmir, R. Kolkowski, J. M. Smith, and S. R. K. Rodriguez, Universal power law decay in the dynamic hysteresis of an optical cavity with non-instantaneous photon-photon interactions (2019), arXiv:1911.00463 [physics.optics].

[49] S. Dufferwiel, F. Li, A. A. P. Trichet, L. Giriunas, P. M. Walker, I. Farrer, D. A. Ritchie, J. M. Smith, M. S. Skolnick, and D. N. Krizhanovskii, Tunable polaritonic molecules in an open microcavity system, Appl. Phys. Lett. 107, 201106 (2015).

[50] D. Sarchi, I. Carusotto, M. Wouters, and V. Savona, Coherent dynamics and parametric instabilities of microcavity polaritons in double-well systems, Phys. Rev. B 77, 125324 (2008).
[51] N. C. Zambon, S. R. K. Rodriguez, A. Lemaitre, A. Harouri, L. L. Gratiet, I. Sagnes, P. St-Jean, S. Ravets, A. Amo, and J. Bloch, Parametric instability in coupled nonlinear microcavities (2019), arXiv:1911.02816 [condmat.mes-hall].

[52] B. Cao, K. W. Mahmud, and M. Hafezi, Two coupled nonlinear cavities in a driven-dissipative environment, Phys. Rev. A 94, 063805 (2016).

[53] W. Casteels and C. Ciuti, Quantum entanglement in the spatial-symmetry-breaking phase transition of a drivendissipative Bose-Hubbard dimer, Phys. Rev. A 95, 013812 (2017).

[54] S. Aldana, C. Bruder, and A. Nunnenkamp, Equivalence between an optomechanical system and a Kerr medium, Phys. Rev. A 88, 043826 (2013).

[55] S. Aldana, C. Bruder, and A. Nunnenkamp, Detection of weak forces based on noise-activated switching in bistable optomechanical systems, Phys. Rev. A 90, 063810 (2014).

[56] L. Papariello, O. Zilberberg, A. Eichler, and R. Chitra, Ultrasensitive hysteretic force sensing with parametric nonlinear oscillators, Phys. Rev. E 94, 022201 (2016).

[57] P. D. Drummond and D. F. Walls, Quantum theory of optical bistability. I. Nonlinear polarisability model, J. Phys. A 13, 725 (1980). 